\title{
Space-Time Coded Systems with Continuous Phase Frequency Shift Keying
}

\author{
Rachel L. Maw and Desmond P. Taylor \\ Department of Electrical and Computer Engineering \\ University of Canterbury, Private Bag 4800, Christchurch, New Zealand \\ rlm57@student.canterbury.ac.nz and taylor@elec.canterbury.ac.nz
}

\begin{abstract}
A space-time (ST) trellis encoded scheme with Mary continuous phase frequency shift keying with a modulation index of $\frac{1}{M}$ (M-CPFSK) is presented. A ST-M-CPFSK system model that incorporates the Rimoldi decomposition of CPFSK is developed. The Rimoldi decomposition separates the non-memory component of CPFSK from the memory component. The latter can be represented as a continuous phase encoder (CPE) and implemented with a ring convolutional encoder. The ST codes used here are linear ring convolutional codes. This allows the ST encoder and the CPE to be combined into one encoder, which can be represented by a single trellis and is easily implemented using delay elements, modulo- $M$ adders and scalar multipliers.
\end{abstract}

\section{INTRODUCTION}

A problem to be considered in designing wireless communication systems is how to reduce the effects of multipath fading. One method is to use diversity techniques that provide replicas of the signal in various forms to the receiver. Space-time coding (STC) [1], [2] uses spatial and temporal diversity. It exploits the diversity available across multiple transmit antennas, to attain good performance, and bandwidth efficiency, on band-limited channels. The multiple paths from the transmit antennas to the receive antenna(s) yield diversity gain, and symbol correlation across the transmit antennas provides coding gain.

Most STC research is based on systems using linear modulations. It was shown in [3] and [4] that continuous phase modulation (CPM) is a good alternative to linear modulation. ST code design with CPM has been investigated in [5] and [6], design rules and general code constructions for specific forms of CPM are derived that guarantee full spatial diversity. An interleaved, externally encoded, ST-CPM system with an iterative soft-output receiver was developed in [7]. A reduced complexity receiver for layered space-time schemes with minimum shift keying (MSK)-like modulations is presented in [8].

Continuous phase frequency shift keying (CPFSK) is a subset of full-response [9] CPM schemes that exhibit the favorable properties of CPM with practical complexity. We have restricted the schemes in this paper to M-ary CPFSK with modulation index $h=\frac{1}{M}$ (M-CPFSK). It was shown in [10] that this modulation index is optimal in terms of energy and bandwidth efficiency. MSK is a special case of M-CPFSK, with $M$ equal to two.

Supported by the Bright Futures Scholarship Scheme administered by the New Zealand Tertiary Education Commission and the Foundation for Research Science and Technology
The Rimoldi [11] model of M-CPFSK separates its memory and non-memory components. A similar decomposition is described in [12]. The memory component of the model is implemented using a convolutional encoder over the ring of integers modulo- $M\left(\mathcal{Z}_{M}\right)$ and is called the continuous phase encoder (CPE) [11]. In this paper a STM-CPFSK system that incorporates the Rimoldi model is developed. The ST codes are convolutional encoders over the same ring as the CPE, which allows the ST encoder and the CPE to be combined into a single trellis encoder.

An introduction to CPM and CPFSK is given in Section II. Section III details the ST-M-CPFSK system with CPFSK decomposed using the Rimoldi model. A feedback-free implementation is then described, it has similar structure to the models developed in [13] and [14]. Examples of ST-M-CPFSK schemes are given in Section IV and simulation results are presented in Section V.

\section{Continuous Phase Modulation}

CPM is a non-linear, constant envelope, modulation scheme. It is suited to transmission over power and bandwidth limited channels, such as mobile satellite channels and land mobile radio [15]. The constant envelope allows the use of low cost, power efficient, non-linear amplifiers. To maintain phase continuity, the modulation has memory. This makes it possible to represent CPM with a trellis.

The general form of a CPM signal [15] is given by

$$
s(t, \boldsymbol{\alpha})=\sqrt{\frac{2 E}{T}} \cos \left(2 \pi f_{o} t+\phi(t, \boldsymbol{\alpha})+\phi_{o}\right) \quad t \geq 0,
$$

where $E$ is the symbol energy, $T$ is the symbol period, $f_{o}$ is the carrier frequency and $\phi_{o}$ is the initial phase offset. The data symbols are $\alpha_{i} \in\{ \pm 1, \pm 3 \ldots \pm(M-1)\}$ for $M$ even. The information carrying phase during the $n$-th symbol interval is

$\phi(t, \boldsymbol{\alpha})=2 \pi h \sum_{i=0}^{n} \alpha_{i} q(t-i T), n T \leq t \leq(n+1) T$,

where the modulation index, $h=\frac{k}{p}$, is chosen to be a ratio of two relatively prime integers, which creates a trellis structure. The phase response, $q(t)$, is the integral of the instantaneous frequency pulse, $g(t)$, and is 0 for $t \leq 0$ and $\frac{1}{2}$ for $t$ greater than the frequency pulse duration $(L T)$. For CPFSK, $L=1$ 


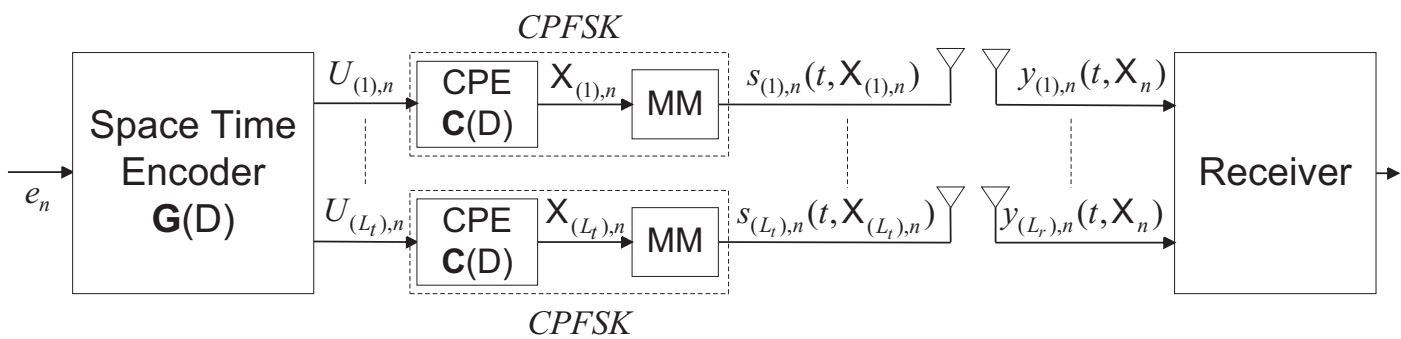

Fig. 1. Block diagram of ST-M-CPFSK

and $g(t)$ is rectangular such that,

$$
q(t)=\left\{\begin{array}{cl}
0 & t \leq 0 \\
\frac{t}{2 T} & 0<t \leq T . \\
\frac{1}{2} & t>T
\end{array} .\right.
$$

\section{A. Ring Convolutional Codes}

In [16] convolutional codes over rings [17] are shown to be natural codes for $M$-ary phase modulation. Let $R=\mathcal{Z}_{M}$ denote a finite commutative ring with multiplicative identity 1. Let $\boldsymbol{R}(D)$ be the ring of rational functions over $R$, where a rational function is the ratio of two polynomials, with the trailing coefficient of the denominator a unit of $R$. The trailing coefficient is the smallest power of $D$ whose coefficient is not equal to zero.

Every rate $\frac{l}{k}$ convolutional code over $R$ can be generated by an $l \times k$ encoding matrix, $\boldsymbol{G}(D) \in \boldsymbol{R}(D)^{l \times k}$. Convolutional codes over rings are natural codes for CPFSK [13], and for more generalized CPM [18].

\section{B. Rimoldi Decomposition}

In [11] Rimoldi developed a model of CPM that separates the memory component (CPE) of CPM from the non-memory component. The model allows the inherent coding of CPM, which is due to the memory required to keep the phase continuous, and the modulation to be considered independently. The CPE is modelled as a linear convolutional encoder. The memoryless component, which follows the CPE is timeinvariant, and is termed the memoryless modulator (MM).

Rimoldi offsets the information carrying phase, $\phi(t, \boldsymbol{\alpha})$ in (2), by $\frac{\pi h(M-1) t}{T}$ to obtain the tilted phase. When the tilted phase is reduced modulo- $2 \pi$ it can be represented by a timeinvariant phase trellis, and is called the physical tilted phase $\left(\tilde{\psi}\left(t, \boldsymbol{X}_{n}\right)\right)$. The vector $\boldsymbol{X}_{n}$ is the input to the MM at time $n T$. It specifies which of $p M^{L}$ physical phase trajectories the modulator should output in the current symbol interval. The CPE generates the input, $\boldsymbol{X}$, to the MM from the modified input data stream, $\boldsymbol{U}$. The $i$-th modified data symbol is calculated from the $i$-th original bipolar symbol as

$$
U_{i}=\left(\frac{\alpha_{i}+(M-1)}{2}\right) \in\{0,1 \ldots(M-1)\} .
$$

For M-CPFSK, the output of the CPE during the $n$-th symbol interval is given by

$$
\boldsymbol{X}_{n}=\left[\begin{array}{ll}
X_{n}^{(1)} & X_{n}^{(2)}
\end{array}\right]=\left[U_{n} V_{n}\right],
$$

$$
V_{n}=\left(V_{n-1}+U_{n-1}\right) \bmod M .
$$

The CPFSK CPE is then described by the generator matrix,

$$
C(D)=\left[\begin{array}{ll}
1 & \frac{D}{1-D}
\end{array}\right] \text {. }
$$

The output of the CPFSK MM during the $n$-th symbol interval is then given by

$$
\begin{gathered}
s\left(t, \boldsymbol{X}_{n}\right)=\sqrt{\frac{2 E}{T}} \cos \left(2 \pi f_{1} t+\tilde{\psi}\left(t, \boldsymbol{X}_{n}\right)\right), \\
\tilde{\psi}\left(t, \boldsymbol{X}_{n}\right)=\left(2 \pi h\left(X_{n}^{(2)}+X_{n}^{(1)} \frac{t-n T}{T}\right)\right) \bmod 2 \pi, \\
n T \leq t \leq(n+1) T,
\end{gathered}
$$

where the offset carrier frequency, $f_{1}$, is defined as

$$
f_{1}=f_{0}-\frac{h(M-1)}{2 T} .
$$

\section{Model of Space-Time Coded M-CPFSK System}

The proposed ST-M-CPFSK system is shown in Fig. 1. It has $L_{t}$ transmit antennas and $L_{r}$ receive antennas. The input data symbols are grouped in frames of $N_{c}$ symbols. Each frame of input symbols is denoted by $e$. The input into the ST encoder during the $n$-th symbol period is an $M$-ary symbol denoted $e_{n}$.

The $\mathrm{CPE}$ is a linear convolutional encoder over $\mathcal{Z}_{M}$. Therefore, it is appropriate to use a ST encoder over the same ring. The ST encoder, $\boldsymbol{G}(D)$, is a rate $\frac{1}{L_{t}}$ convolutional encoder. This provides a natural means to obtain $L_{t}$ output streams. The output of the ST encoder is given by,

$$
\boldsymbol{U}=\boldsymbol{e} \boldsymbol{G}(D) \text {. }
$$

The output during the $n$-th symbol period is the $L_{t}$ dimensional vector,

$$
\boldsymbol{U}_{n}=\left[\begin{array}{llll}
U_{(1), n} & U_{(2), n} & \ldots & U_{\left(L_{t}\right), n}
\end{array}\right] .
$$

Each element of $\boldsymbol{U}_{n}$ is an M-ary symbol. The output of the ST encoder is split into $L_{t}$ streams,

$$
\boldsymbol{U}=\left[\begin{array}{llll}
\boldsymbol{U}_{(1)} & \boldsymbol{U}_{(2)} & \ldots & \boldsymbol{U}_{\left(L_{t}\right)}
\end{array}\right],
$$

where the $i$-th stream is given by

$$
\boldsymbol{U}_{(i)}=\left[\begin{array}{llll}
U_{(i), 0} & U_{(i), 1} & \ldots & U_{(i), N_{c}-1}
\end{array}\right]^{T}, i=1,2, \ldots, L_{t} .
$$




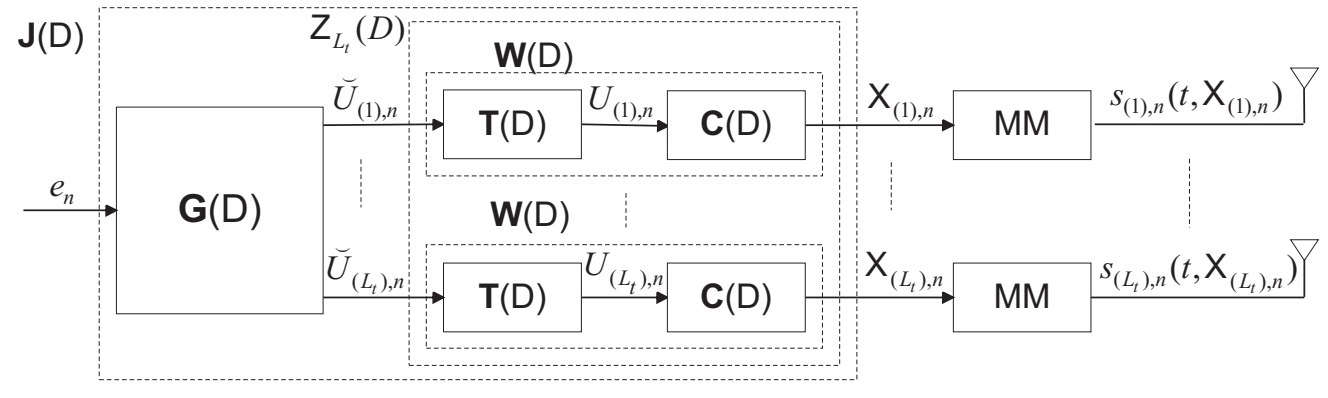

Fig. 2. Block diagram of ST-M-CPFSK transmitter with CPE precoder

The output from the $i$-th CPE during the $n$-th symbol period is then

$$
\boldsymbol{X}_{(i), n}=U_{(i), n} \boldsymbol{C}(D)
$$

a vector of two M-ary elements. The signal $\left(s_{(i), n}\left(t, \boldsymbol{X}_{(i), n}\right)\right)$ that is transmitted from antenna $i$ in the $n$-th symbol interval is found using (8) and (9).

Each receive antenna receives a faded superposition of the $L_{t}$ simultaneously transmitted signals corrupted by additive white Gaussian noise (AWGN). The fading is assumed to be flat Rayleigh fading. The random path gain between transmit antenna $i$ and receive antenna $j, m_{i, j}(t)$, is an independent complex Gaussian random variable with zero mean and a variance of $\frac{1}{2}$ per dimension. The fading is slow, such that the $L_{t} \times L_{r}$ fading coefficients are constant during a frame, but vary from frame to frame. The AWGN noise component, $n_{j}(t)$, is an independent zero-mean complex Gaussian random process, with power spectral density $N_{0}$. The received signal on antenna $j$ is then

$$
y_{(j)}(t, \boldsymbol{X})=\sum_{i=1}^{L_{t}} m_{i, j} s_{(i)}\left(t, \boldsymbol{X}_{(i)}\right)+n_{j}(t), 0 \leq t \leq N_{c} T .
$$

The received information is decoded using the Viterbi algorithm [19] and the trellis of the overall encoder. The maximum likelihood metric for the ST-M-CPFSK system assuming perfect channel state information is given [6] by

$$
\begin{aligned}
& M(y(t, \boldsymbol{X}) / \hat{\boldsymbol{X}}) \\
= & -\sum_{j=1}^{L_{r}} \int_{0}^{N_{c} T}\left|y_{(j)}(t, \boldsymbol{X})-\sum_{i=1}^{L_{t}} m_{i, j} s_{(i)}\left(t, \hat{\boldsymbol{X}}_{(i)}\right)\right|^{2} d t,
\end{aligned}
$$

where $s_{(i)}\left(t, \hat{\boldsymbol{X}}_{(i)}\right)$ is the hypothesized transmitted signal from transmit antenna $i$. The metric can be rewritten as a sum over $N_{c}$ symbol intervals as

$$
\begin{aligned}
& M(y(t, \boldsymbol{X}) / \hat{\boldsymbol{X}})=-\sum_{n=0}^{N_{c}-1} \sum_{j=1}^{L_{r}} \int_{n T}^{(n+1) T} \\
& \left|y_{(j), n}\left(t, \boldsymbol{X}_{\boldsymbol{n}}\right)-\sum_{i=1}^{L_{t}} m_{i, j} s_{(i), n}\left(t, \hat{\boldsymbol{X}}_{(i), n}\right)\right|^{2} d t .
\end{aligned}
$$

\section{A. Code Search Model}

A catastrophic encoder can produce an infinite number of output errors given a finite number of input errors [20]. Therefore, systems with overall catastrophic encoders are to be avoided. When CPFSK is combined with a catastrophic external encoder it does not necessarily result in an overall catastrophic encoder [21]. Similarly, a non-catastrophic encoder combined with CPFSK may produce an overall catastrophic system. This behavior is due to the external encoder interacting with feedback from the CPE. The CPE feedback can be removed using a precoder [20], reducing the search for ST encoders to those that are non-catastrophic. by

The precoder generator matrix [13] for M-CPFSK is given

$$
\boldsymbol{T}(D)=[1-D] .
$$

This precoder is a scrambler [22], [13]. $\boldsymbol{T}(D)$ is cascaded with the CPE, to create a feedback-free CPE (FF-CPE) with generator,

$$
\begin{aligned}
\boldsymbol{W}(D) & =\boldsymbol{T}(D) \boldsymbol{C}(D) \\
& =\left[\begin{array}{ll}
1-D & D
\end{array}\right] .
\end{aligned}
$$

Non-feedback ST encoders have been considered in this paper. The concatenation of the ST encoder and the feedback-free CPE results in a feedback-free space-time continuous phase encoder (FF-ST-CPE). This will later allow a non-catastrophic external error control encoder to be concatenated with the system to produce an overall non-catastrophic encoder. Fig. 2 shows the block diagram of the modified transmitter.

\section{B. Combined Encoder}

Each encoder in Fig. 2 is a linear convolutional encoder over $\mathcal{Z}_{M}$. Therefore, the entire encoding system can be combined into a single linear convolutional encoder over $\mathcal{Z}_{M}$.

The $L_{t}$ feedback free CPEs, $\boldsymbol{W}(D)$, are combined into a rate $\frac{1}{2}$ encoder with an $L_{t} \times 2 L_{t}$ generator matrix given by,

$$
\boldsymbol{Z}_{L_{t}}(D)=\left[\begin{array}{cccccc}
1-D & D & 0 & 0 & \cdots & 0 \\
0 & 0 & 1-D & D & \ddots & \\
\vdots & \ddots & \ddots & \ddots & \ddots & \\
0 & & 0 & 0 & 1-D & D
\end{array}\right] \text {. }
$$


The input into $Z_{L_{t}}(D)$ during the $n$-th symbol period is the $L_{t}$ dimensional vector,

$$
\begin{aligned}
\breve{\boldsymbol{U}}_{n}= & {\left[\breve{U}_{(1), n} \breve{U}_{(2), n} \ldots \breve{U}_{\left(L_{t}\right), n}\right], } \\
& \breve{U}_{(i), n} \in\{0,1, \ldots,(M-1)\}, i=1,2, \ldots, L_{t} .
\end{aligned}
$$

The output is the $2 L_{t}$ dimensional vector,

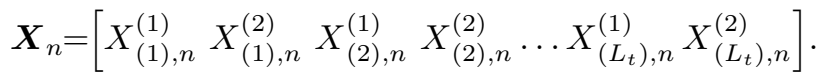

The ST encoder $\boldsymbol{G}(D)$ can be cascaded with $\boldsymbol{Z}_{L_{t}}(D)$ to form a FF-ST-CPE over $\mathcal{Z}_{M}$ with generator matrix,

$$
\boldsymbol{J}(D)=\boldsymbol{G}(D) \boldsymbol{Z}_{L_{t}}(D) .
$$

The input into $\boldsymbol{J}(D)$ during the $n$-th symbol period is the symbol $e_{n}$ and the output is the $2 L_{t}$ dimensional vector $\boldsymbol{X}_{n}$ of (23). The throughput of the overall ST-M-CPFSK system is one $M$-ary symbol of information per interval.

\section{ST-M-CPFSK SYSTEM EXAMPLES}

Delay diversity [1] is a simple ST code that has been used with MSK in [4]. It can be employed with any number of transmit and receive antennas. The delay diversity ST encoder for a system with $L_{t}$ transmit antennas has the generator matrix,

$\boldsymbol{G}_{\mathbf{2}^{L_{t^{-1}}, \mathbf{2}^{L_{t}-\mathbf{2}}, . ., \mathbf{1}}}(D)=\left[\begin{array}{lllll}D^{\left(L_{t}-1\right)} & D^{\left(L_{t}-2\right)} & \cdots & D & 1\end{array}\right]$.

Delay diversity ST codes always achieve full spatial diversity in ST-CPM systems [5].

The overall encoder of a delay diversity ST-M-CPFSK system with two transmit antennas is easily seen to have the generator matrix,

$$
\begin{aligned}
\boldsymbol{J}(D) & =\boldsymbol{G}_{\mathbf{2 , 1}}(D) \boldsymbol{Z}_{2}(D) \\
& =\left[\begin{array}{ll}
D & 1
\end{array}\right]\left[\begin{array}{llll}
1-D & D & 0 & 0 \\
0 & 0 & 1-D & D
\end{array}\right] \\
& =\left[\begin{array}{llll}
D-D^{2} & D^{2} & 1-D & D
\end{array}\right] .
\end{aligned}
$$

An implementation of this is shown in Fig. 3. The encoder is on the ring of integers modulo- $M\left(\mathcal{Z}_{M}\right)$.

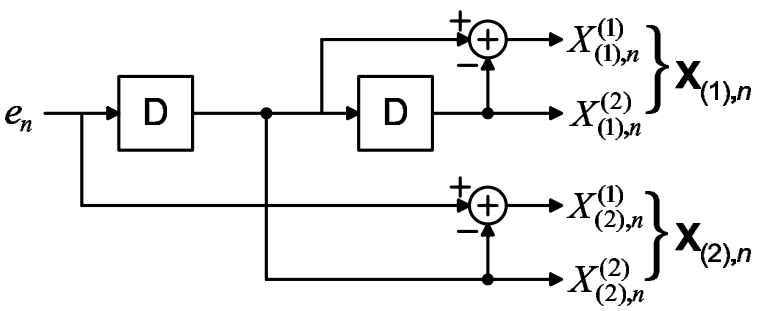

Fig. 3. Overall encoder of delay diversity ST-M-CPFSK with $L_{t}=2$

The overall trellis for the 2 transmit antenna, delay diversity ST system with MSK (2-CPFSK) is shown in Fig. 4. $S_{1}$ and $S_{2}$ are the states of the delay elements in the encoder. The branches are labelled with the corresponding input and output symbols for that transition, $e_{n}\left(X_{(1), n}^{(1)} X_{(1), n}^{(2)} X_{(2), n}^{(1)} X_{(2), n}^{(2)}\right)$. There is a similar trellis in [4] used for data detection.

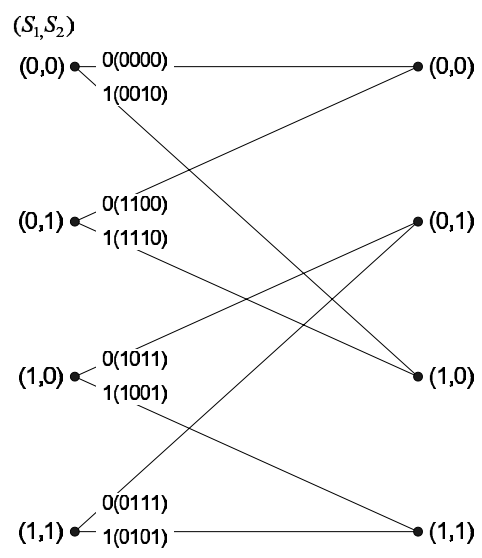

Fig. 4. Trellis diagram of delay diversity ST-2-CPFSK with $L_{t}=2$

The 2 transmit antenna, delay diversity ST-4-CPFSK system is implemented using the encoder shown in Fig. 3, with modulo-4 adders. The trellis for the system has 16 states. Similarly, the 8-CPFSK system is implemented on $\mathcal{Z}_{8}$. For delay diversity ST-M-CPFSK systems, the constraint length of the overall encoder is equal to $L_{t}$ and the number of states in the overall combined trellis is $M^{L_{T}}$. Therefore, delay diversity ST-8-CPFSK with two transmit antennas has a 64 state trellis.

The ST encoder $\boldsymbol{G}_{\mathbf{5 , 7}}(D)$ is rate $\frac{1}{2}$, with generator matrix,

$$
\boldsymbol{G}_{\mathbf{5 , \boldsymbol { 7 }}}(D)=\left[\begin{array}{ll}
D^{2}+1 & D^{2}+D+1
\end{array}\right] .
$$

In this paper, rate $\frac{1}{L_{t}} \mathrm{ST}$ encoders are used, therefore $\boldsymbol{G}_{\mathbf{5 , 7}}(D)$ is used in a two transmit antenna system. The resulting STM-CPFSK generator is given by

$$
\begin{aligned}
\boldsymbol{J}(D) & =\boldsymbol{G}_{\mathbf{5 , 7}}(D) \boldsymbol{Z}_{2}(D) \\
& =\left[\begin{array}{llll}
-D^{3}+D^{2}-D+1 & D^{3}+D & -D^{3}+1 & D^{3}+D^{2}+D
\end{array}\right] .
\end{aligned}
$$

The ST encoder, $\boldsymbol{G}_{\mathbf{5 , 6 , 7}}(D)$, is rate $\frac{1}{3}$ and has the generator

$$
\boldsymbol{G}_{\mathbf{5 , 6 , 7}}(D)=\left[\begin{array}{lll}
D^{2}+1 & D^{2}+D & D^{2}+D+1
\end{array}\right] .
$$

It is used in a three transmit antenna system. The combined ST-M-CPFSK generator is given by

$$
\begin{aligned}
& \boldsymbol{J}(D)=\boldsymbol{G}_{\mathbf{5}, \mathbf{6}, \mathbf{7}}(D) \boldsymbol{Z}_{3}(D) \\
& =\left[\begin{array}{lll}
-D^{3}+D^{2}-D+1 & D^{3}+D & -D^{3}+D
\end{array}\right. \\
& \left.D^{3}+D^{2} \quad-D^{3}+1 \quad D^{3}+D^{2}+D\right] .
\end{aligned}
$$

For $\boldsymbol{G}_{\mathbf{5 , 7}}(D)$ and $\boldsymbol{G}_{\mathbf{5 , 6 , 7}}(D)$, any value of $M$ can be used for the modulation. The overall encoders are linear over $\mathcal{Z}_{M}$ and both have a constraint length of three. Thus, they have $M^{3}$ trellis states. These encoders also achieve full diversity [5] in ST-CPM systems.

\section{Simulation Results And Discussion}

Fig. 5 shows simulation results of MSK schemes with delay diversity ST codes $\boldsymbol{G}_{\mathbf{2 , 1}}, \boldsymbol{G}_{\mathbf{4 , 2 , 1}}$ and $\boldsymbol{G}_{\mathbf{8 , 4 , 2 , 1}}$ that have 2, 3 and 4 transmit antennas respectively, and 1 receive antenna. An increased diversity gain corresponding to an increased number 
TABLE I

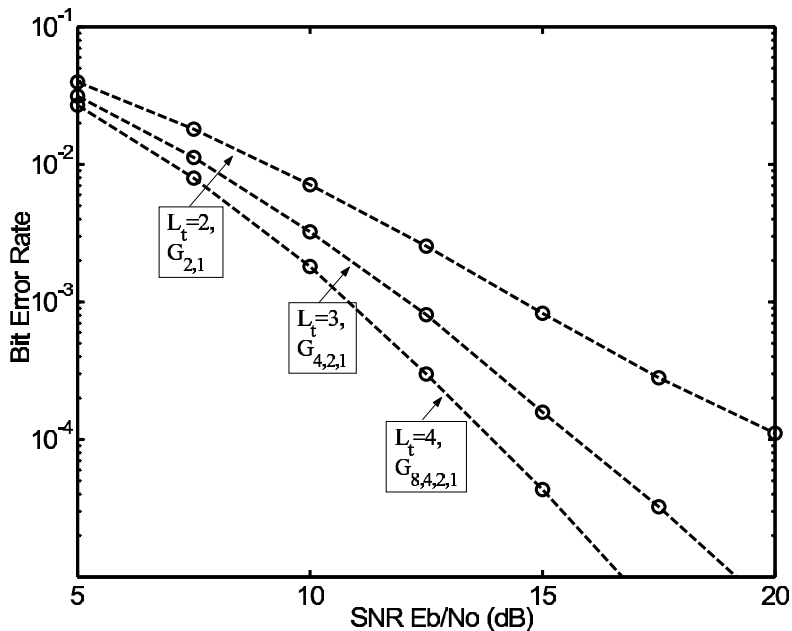

Fig. 5. Performance results of delay diversity ST codes with MSK, $L_{r}=1$

of transmit antennas is observed. The number of states in the decoding trellis is 4,8 and 16 for the 2, 3 and 4 transmit antenna codes respectively. $M$ is equal to 2 for each system, hence each system's code rate is 1 bit per symbol.

For linear modulations the design criteria [23] for optimal ST codes depends on the value of $\rho L_{r}$, where $\rho$ is the rank of the codeword difference matrix and $\rho \leq L_{t}$. If $\rho L_{r} \leq 3$, the rank and the minimum determinant [1] of the matrix are the important parameters for code design. If $\rho L_{r}>3$, the trace of the matrix or equivalently the normalized minimum squared Euclidean distance $\left(d_{\min }^{2}\right)$ should be used as the design criteria.

The performance of a ST-CPM system has a direct analogy to the performance of ST coded linear modulation [6]. The results derived for linear modulations can be applied to CPM, by considering the signal matrix,

$\boldsymbol{S}=\left[\begin{array}{ccc}\int_{0}^{N_{c} T}\left|\triangle_{1}(t)\right|^{2} d t & \cdots & \int_{0}^{N_{c} T} \triangle_{1}^{*}(t) \triangle_{L_{t}}(t) d t \\ \vdots & \vdots & \vdots \\ \int_{0}^{N_{c} T} \triangle_{1}(t) \triangle_{L_{t}}^{*}(t) d t & \cdots & \int_{0}^{N_{c} T}\left|\triangle_{L_{t}}(t)\right|^{2} d t\end{array}\right]$,

where $\triangle_{i}(t)=s(t, \boldsymbol{X})-s(t, \hat{\boldsymbol{X}})$ is the continuous time difference between the transmitted and the decoded signal from the $i$-th transmit antenna, instead of the codeword difference matrix. The rank, determinant and trace or $d_{\min }^{2}$ criteria thus hold for ST-M-CPFSK systems, when used with (31).

The encoders $\boldsymbol{G}_{\mathbf{5 , 7}}$ and $\boldsymbol{G}_{\mathbf{2}, \mathbf{1}}$ have full spatial diversity and $L_{t}=2$, hence $\rho$ is equal to 2 . Therefore, when $L_{r} \geq 2$, $d_{\min }^{2}$ dominates the relative performance of these encoders. Similarly, $\boldsymbol{G}_{\mathbf{5 , 6 , 7}}$ and $\boldsymbol{G}_{\mathbf{4 , 2 , \mathbf { 1 }}}$ have full diversity and $\rho=L_{t}=$ 3. Again, for $L_{r} \geq 2, d_{\min }^{2}$ is the dominant factor for the codes' performance. For the 4 transmit antenna delay diversity code $\boldsymbol{G}_{\mathbf{8 , 4 , 2}, \mathbf{1}} \rho$ is equal to 4 , hence for any number of receive antennas $d_{\mathrm{min}}^{2}$ is the dominant factor for its performance.

The normalized minimum squared Euclidean distance between space-time encoded M-CPFSK sequences can be calculated using the combined trellis. The output of the combined
VALUES OF $d_{\min }^{2}$ FOR ST-M-CPFSK SYSTEMS

\begin{tabular}{|c|c|c|c|c|c|}
\hline STC & M-CPFSK & $L_{t}$ & Trellis States & Code Rate & $d_{\min }^{2}$ \\
\hline$G_{2,1}$ & MSK & 2 & 4 & 1 & 2.00 \\
\hline$G_{5,7}$ & MSK & 2 & 8 & 1 & 5.00 \\
\hline$G_{4,2,1}$ & MSK & 3 & 8 & 1 & 2.00 \\
\hline$G_{5,6,7}$ & MSK & 3 & 8 & 1 & 4.67 \\
\hline$G_{8,4,2,1}$ & MSK & 4 & 16 & 1 & 2.00 \\
\hline$G_{2,1}$ & 4-CPFSK & 2 & 16 & 2 & 1.45 \\
\hline$G_{5,7}$ & 4-CPFSK & 2 & 64 & 2 & 3.27 \\
\hline$G_{4,2,1}$ & 4-CPFSK & 3 & 64 & 2 & 1.45 \\
\hline$G_{5,6,7}$ & 4-CPFSK & 3 & 64 & 2 & 3.15 \\
\hline$G_{8,4,2,1}$ & 4-CPFSK & 4 & 256 & 2 & 1.45 \\
\hline$G_{2,1}$ & 8-CPFSK & 2 & 64 & 3 & 0.60 \\
\hline$G_{5,7}$ & 8-CPFSK & 2 & 512 & 3 & 1.35 \\
\hline$G_{4,2,1}$ & 8-CPFSK & 3 & 512 & 3 & 0.60 \\
\hline$G_{5,6,7}$ & 8-CPFSK & 3 & 512 & 3 & 1.30 \\
\hline
\end{tabular}

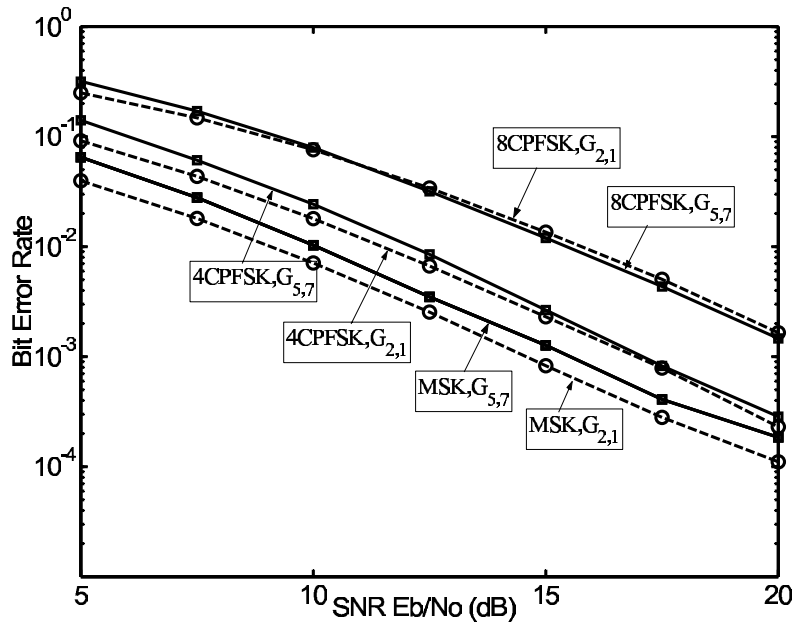

Fig. 6. Performance results of $L_{t}=2, L_{r}=1$ ST-M-CPFSK systems

trellis is the input to the memoryless modulators, of which $d_{\mathrm{min}}^{2}$ is a function of for CPFSK [24], [25]. The value of $d_{\mathrm{min}}^{2}$ for various ST-M-CPFSK systems are given in Table I. For a given value of $M$, the delay diversity $\mathrm{ST}$ codes have the same $d_{\text {min }}^{2}$ for 2, 3 and 4 transmit antennas. For a given modulation and number of transmit antennas, $\boldsymbol{G}_{\mathbf{5 , 7}}$ and $\boldsymbol{G}_{\mathbf{5 , 6 , 7}}$ have larger $d_{\text {min }}^{2}$ compared to the delay diversity codes. In general, when the overall system rate is increased through $M, d_{\text {min }}^{2}$ decreases.

Fig. 6 shows simulations of various 2 transmit and 1 receive antenna ST coded systems. The MSK, 4-CPFSK and 8-CPFSK systems have code rates of 1,2 and 3 bits per symbol, respectively. As the code rate and the modulation alphabet increases, the performance degrades. The diversity gain, which can be determined by the slope of the curves is the same for each system, as was expected. The $\boldsymbol{G}_{\mathbf{5 , 7}}$ ST codes perform worse than the delay diversity ST codes for MSK and 4CPFSK. The delay diversity 8-CPFSK scheme has similar performance to that of the corresponding $\boldsymbol{G}_{\mathbf{5 , 7}}$ system. For each modulation, the delay diversity code has a value of $d_{\min }^{2}$ that is less than half the value for the $\boldsymbol{G}_{\mathbf{5 , 7}}$ code. This shows 


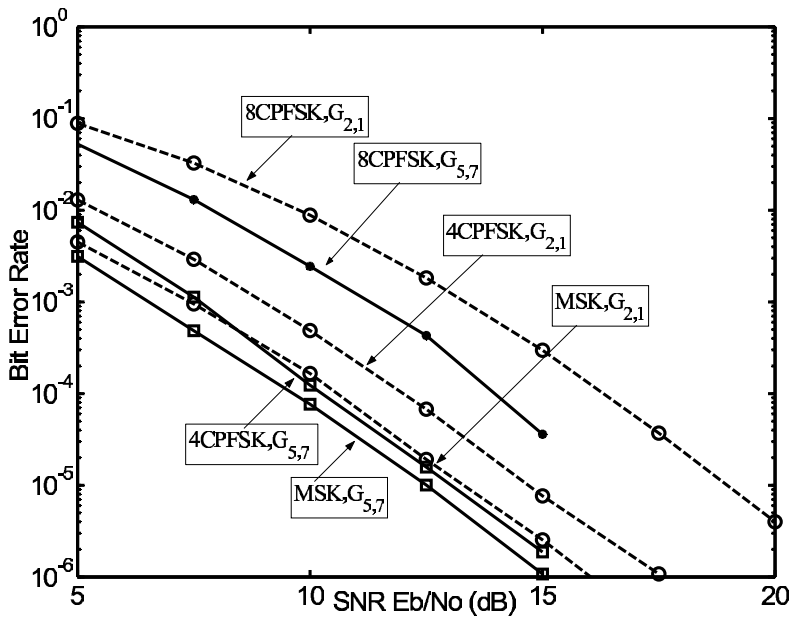

Fig. 7. Performance results of $L_{t}=2, L_{r}=2$ ST-M-CPFSK systems

that $d_{\min }^{2}$ is not the dominant parameter determining system performance for this configuration.

When the number of receive antennas is increased to 2 , $d_{\min }^{2}$ between the encoded sequences becomes the most important parameter for the comparative performance of these schemes. This is illustrated in Fig. 7, which shows ST coded systems with 2 transmit and 2 receive antennas. The $\boldsymbol{G}_{\mathbf{5}, \mathbf{7}}$ systems, which have better minimum squared Euclidean distance, outperform the corresponding delay diversity systems. The difference in performance is more pronounced with four receive antennas.

\section{Vi. Conclusions}

In this paper we presented a ST-M-CPFSK model that allows the ST encoder and the CPE to be combined into a single trellis encoder. The overall encoder is a linear convolutional encoder over the ring of integers modulo- $M$. The model is easily extended to incorporate external error correction encoding.

The combined trellis is used by the receiver in the Viterbi algorithm. The trellis can also be used to find the minimum squared Euclidean distance of the ST coded scheme. For CPFSK $d_{\min }^{2}$ is a function of the input to the memoryless modulator.

Using the design criterion in [5], [6] codes with full spatial diversity can be found. For all schemes except those that have 2 or 3 transmit antennas and 1 receive antenna, the best codes are then found by comparing $d_{\min }^{2}$. For schemes with 4 or more receive antennas it is not necessary to find codes with full spatial diversity.

\section{REFERENCES}

[1] V. Tarokh, N. Seshadri, and A. R. Calderbank, "Space-time codes for high data rate wireless communication: Performance analysis and code construction," IEEE Trans. Inform. Theory, vol. 44, no. 2, pp. 744-765, Mar. 1998.

[2] J.-C. Guey, M. P. Fitz, M. R. Bell, and W.-Y. Kuo, "Signal design for transmitter diversity wireless communication systems over Rayleigh fading channels," in Proc. Veh. Tech. Conf., vol. 46, Atlanta, GA, 1996, pp. 136-140.

[3] X. Zhang and M. P. Fitz, "Constant envelope space-time modems," in Proc. Veh. Tech. Conf., vol. 3, Oct. 2003, pp. 1772-1776.

[4] J. K. Cavers, "Space-time coding using MSK," in Proc. Veh. Tech. Conf., vol. 1 , Sept 2002, pp. 401-405.

[5] X. Zhang and M. P. Fitz, "Space-time code design with CPM transmission," in Proc. Int. Symp. Inform. Theory, Washington, DC, Jun. 2001, p. 327.

[6] - "Space-time code design with continuous phase modulation," IEEE J. Select. Areas Commun., vol. 21, no. 5, pp. 783-792, Jun. 2003.

[7] — "Soft-output demodulator in space-time-coded continuous phase modulation," IEEE Trans. Signal Processing, vol. 50, no. 10, pp. 2589 2598, Oct. 2002.

[8] W. Zhao and G. B. Giannakis, "Reduced complexity receivers for layered space-time CPM," IEEE Trans. Wireless Commun., vol. 4, no. 2, pp. 574-582, Mar. 2005.

[9] T. Aulin and C.-E. W. Sundberg, "Continuous phase modulation - Part I: Full response signalling," IEEE Trans. Commun., vol. 29, no. 3, pp. 196-209, Mar. 1981.

[10] B. E. Rimoldi, "Design of coded CPFSK modulation systems for bandwidth and energy efficiency," IEEE Trans. Commun., vol. 37, no. 9, pp. 897-905, Sep. 1989.

[11] — "A decomposition approach to CPM," IEEE Trans. Inform. Theory, vol. 34, no. 2, pp. 260-270, Mar. 1988.

[12] J. Huber and W. Liu, "Convolutional codes for CPM using the memory of the modulation process," in Proc. Global Telecommun. Conf., vol. 3 , 1987, pp. $1687-1691$

[13] R. H.-H. Yang and D. P. Taylor, "Trellis-coded continuous-phase frequency-shift keying with ring convolutional codes," IEEE Trans. Inform. Theory, vol. 40, no. 4, pp. 1057-1067, Jul. 1994.

[14] A. Griffin and D. P. Taylor, "Coding CPFSK for differential demodulation," IEEE Trans. Commun., vol. 48, pp. 721-724, May 2000.

[15] J. B. Anderson, T. Aulin, and C.-E. W. Sundberg, Digital Phase Modulation. NY: Plenum, 1986.

[16] J. L. Massey and T. Mittelholzer, "Convolutional codes over rings," in Proc. 4th Joint Swedish-USSR Workshop on Info. Th., Gotland, Sweden, Aug.-Sep. 1989, pp. 14-18.

[17] _ "Systematicity and rotational invariance of convolutional codes over rings," in Proc. 2nd Int. Workshop on Algebraic and Combinatorial Coding Th., Leningrad, Sep. 1990, pp. 154-159.

[18] B. E. Rimoldi and Q. Li, "Coded continuous phase modulation using ring convolutional codes," IEEE Trans. Commun., vol. 43, no. 11, pp. 2714-2720, Nov. 1995.

[19] G. D. Forney, Jr., "The Viterbi algorithm," Proc. IEEE, vol. 61, no. 3, pp. 268-277, Mar. 1973.

[20] F. Morales-Moreno, W. Holubowicz, and S. Pasupathy, "Optimization of trellis coded TFM via matched codes," IEEE Trans. Commun., vol. 42, no. 2/3/4, pp. 1586-1594, Feb./Mar./Apr. 1994.

[21] F. Morales-Moreno and S. Pasupathy, "Structure, optimization, and realization of FFSK trellis codes," IEEE Trans. Inform. Theory, vol. 34, no. 4, pp. 730-751, Jul. 1988.

[22] G. D. Forney, Jr., "Convolutional codes I: Algebraic structure," IEEE Trans. Inform. Theory, vol. 16, no. 6, pp. 720-738, Nov. 1970.

[23] Z. Chen, J. Yuan, and B. Vucetic, "An improved space-time trellis coded modulation scheme on slow Rayleigh fading channels," in Proc. Int. Conf. Commun., vol. 4, Jun. 2001, pp. 1110-1116.

[24] J. B. Anderson and D. P. Taylor, "A bandwidth-efficient class of signal space codes," IEEE Trans. Inform. Theory, vol. 24, pp. 703-712, Nov. 1978.

[25] B. E. Rimoldi, "Exact formula for the minimum squared Euclidean distance of CPFSK," IEEE Trans. Commun., vol. 39, no. 9, pp. 12801282, Sep. 1991. 